\title{
Search for isotropic microwave radiation from electron beam in the atmosphere
}

\author{
T. Yamamoto ${ }^{* a}$, I. S. Ohota ${ }^{a}$, Y. Inome ${ }^{a}$, D. Ikeda ${ }^{b}$, H. Sagawa ${ }^{b}$, S. Ogio ${ }^{c}$, T. Sako ${ }^{d}$, T. \\ Shibata ${ }^{e}$, J. N. Mattherws ${ }^{f}$ \\ E-mail: tokonatu@konan-u.ac.jp \\ ${ }^{a}$ Faculty of Science and Engineering, Konan University, Kobe 658-8501, Japan; \\ ${ }^{b}$ Institute for Cosmic Ray Research, University of Tokyo, Kashiwa, Chiba 277-8582, Japan; \\ ${ }^{c}$ Graduate School of Science, Osaka City University, Osaka 558-8585, Japan; \\ ${ }^{d}$ Solar-Terrestrial Environment Laboratory, Nagoya University, Nagoya 464-8601, Japan; \\ ${ }^{e}$ High Energy Accelerator Research Organization (KEK), Tsukuba 305-0801, Japan; \\ ${ }^{f}$ University of Utah, Salt Lake City, UT 4112-0830, USA;
}

We report a search for $12.5 \mathrm{GHz}$ microwave radiation from electron beams in the atmosphere. Ultrahigh-energy cosmic rays (UHECRs) are currently observed indirectly through extensive air showers (EASs) by particle detectors on the ground or fluorescence detectors using a remote sensing method. If isotropic radiation of microwave from EAS is detected, it can be used for future observation of the UHECR based on a remote sensing method just like fluorescence detector with $100 \%$ duty cycle like particle detectors. Week attenuation in the atmosphere is another advantage to measure microwave radiation. To study microwave radiation from EAS, we used Electron Light Source (ELS) located at the Telescope Array Observatory in Utah, USA. The ELS emitted electron beams vertically into the atmosphere. Energy of the electron in the beam is 40 $\mathrm{MeV}$ which is similar to that in the EAS. About $10^{9}$ electrons are contained in a beam, which is equivalent to the shower maximum of an air shower created from $10^{18} \mathrm{eV}$ cosmic ray. The beam is triangular pulse of which the base is $20 \mathrm{~ns}$. Commercial equipment for the satellite television are utilized for the microwave detection system. $1.2 \mathrm{~m}$ diameter parabola with $12.5 \mathrm{GHz}$ receiver which measures vertical and horizontal polarizations is fixed on a concrete pad located at $88 \mathrm{~m}$ away from the electron beam. 1797 beam shots were observed and no microwave signal has been detected. In this contribution we will report details of this detector, its calibration and obtained upper-limit on the intensity of isotropic radiation of $12.5 \mathrm{GHz}$ microwave.

The 34th International Cosmic Ray Conference,

30 July- 6 August, 2015

The Hague, The Netherlands

\footnotetext{
* Speaker.
} 


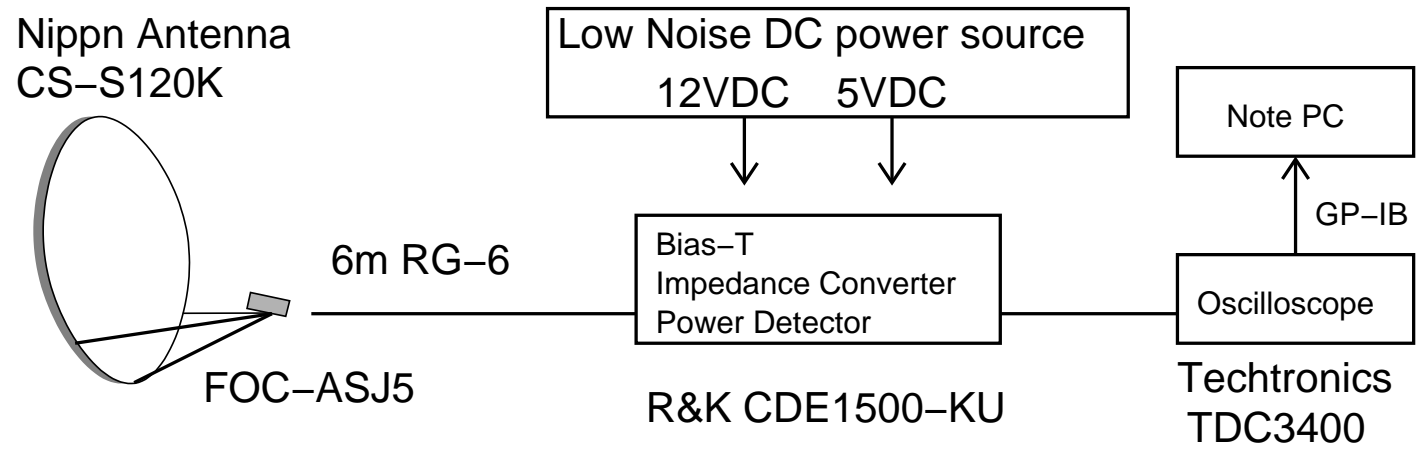

Figure 1: Schematic illustration of observation system.

\section{Introduction}

Observatory of the UHECR has been expanding continually since statistics of the CR data still limit to find its origin. A $3000 \mathrm{~km}^{2}$ of water-Cherenkove-detector array had been constructed on a pampa in Argentina so called the Pierre Auger observatory [1]. The Auger is the largest cosmic-ray detector and have measured UHECR with unprecedented precision. On the northern hemisphere, the $700 \mathrm{~km}^{2}$ of scintillator array had been constructed on a desert area in Utah USA so called the Telescope Array observatory (TA) [2]. Recently this observatory was approved to expand to similar size of Auger. These observatories found hints of anisotropies in the UHECR sky although statistics still limit to make a clear conclusion [3].

UHECRs are detected indirectly using particle detectors such as water Cherenkov and scintillator detectors so called surface detector (SD). Atmospheric-fluorescence detector (FD) is also used for the indirect measurement. The SDs are spread over a wide area on the ground and measure number of secondary particles from the extensive air showers (EASs) which is created from UHECRs. An advantage of SDs is that they provide stable observation at all times, whereas a disadvantage is that they must be spread over a large area. In addition, SDs can only observe a single slice of an air shower. FDs use a remote-sensing technique to observe the isotropic radiation of ultra-violet photons emitted by air showers. This method allows us to observe the lateral distribution of secondary particles but works only on clear, dark nights.

Air showers radiate microwaves via various processes such as geo-synchrotron radiation caused by the geomagnetic field and Cherenkov radiation with Askaryan effect. These processes cause polarized microwaves to be radiated in the forward direction along the shower axis allowing continuous observation as SDs. Apart from such radiation in the forward direction, isotropic microwave radiation may allow entire air showers to be observed remotely using the same approach as FDs. If the isotropic microwave radiation is sufficiently intense to be detected, it can be exploited by nextgeneration techniques to observe UHECRs, thereby allowing us to make unbroken observation of entire air shower.

One of the processes of the isotropic microwave radiation is molecular bremsstrahlung radiation (MBR). The secondary electrons in an air shower ionize atmospheric molecules creating low energy electrons. The low energy electrons are scattered by the molecules radiating unpolarized 

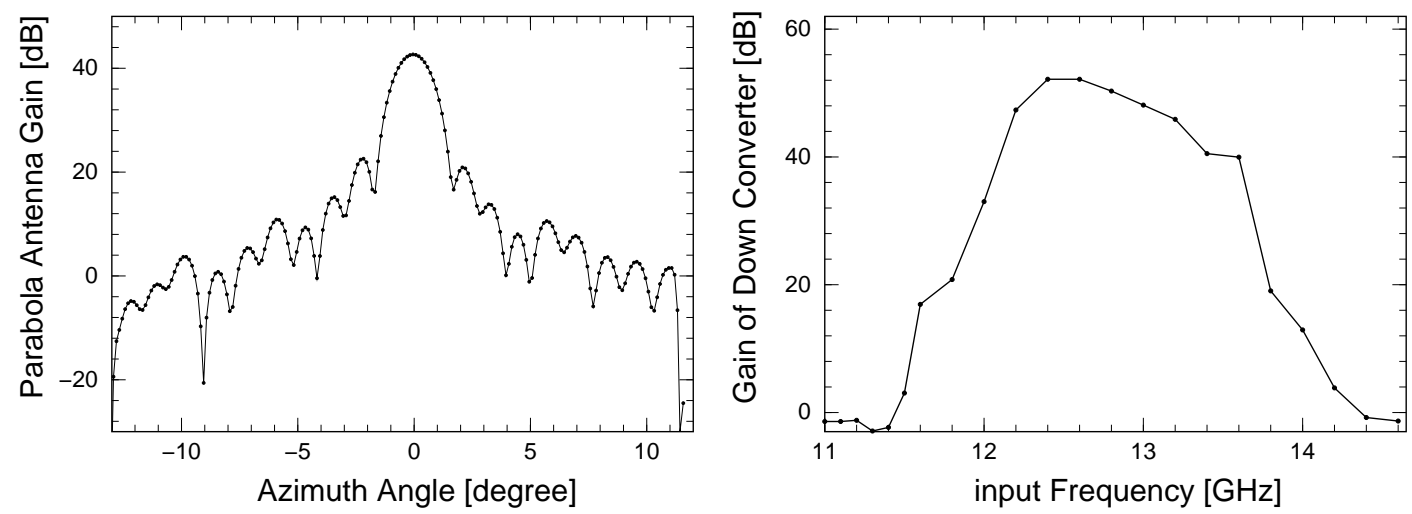

Figure 2: Results of calibrations of microwave receiver. The left panel shows the antenna gain and the pattern of the parabola and feed horn. The right panel shows the gain of the down converter as a function of frequency of the input microwaves. The results of these measurements are consistent with the values in the catalog of the detectors. These measurements were performed at the Microwave Energy Transmission Laboratory in Kyoto University [5].

microwave. Previous study experimentally confirmed MBR using electron accelerators [4]. The results from this experiment indicates sufficient intensity of the isotropic microwave radiation to use observation of UHECRs. Following this study, several experiments have been performed to detect microwave from extensive air-showers. Some of these experiments inject electron beams from accelerator into anechoic chamber. These experiments suffered from background noises caused by accelerator and the beam itself by Cherenkov radiation. The others are measuring microwave from extensive air showers with cosmic ray detectors.

There is a unique device in the TA observatory which emits electron beam into the atmosphere to simulate extensive air showers. We used this ELS for a measurement of the isotropic microwave radiation from air shower. Based on this measurements, an upper-limit on the intensity of 12.5 $\mathrm{GHz}$ microwave radiation was estimated. In this contribution we report this measurement of the isotropic microwave radiation from electron beams.

\section{Experiment}

We developed a microwave observation system to measure the isotropic radiation from EAS. Devices for the satellite television receivers are utilized in this observation system as shown in Figure 1. An off-axis parabola (Nippon Antenna CS-S120K) with diameter of $1.32 \mathrm{~m}$ and 1.22 $\mathrm{m}$ in major and minor axes respectively is used. This parabola is equivalent to a 1.2-m-diameter circular reflector. A feed-horn receiver with a bandwidth of $12.25 \sim 12.75 \mathrm{GHz}$ (Nippon Antenna FOC-ASJ5) is mounted at the focal point. To measure vertical and horizontal polarization, the receiver has two channels. The microwave signal is converted down to $0.95 \sim 1.45 \mathrm{GHz}$ by a local oscillator with a frequency of $11.3 \mathrm{GHz}$. The signal from the down converter goes through an amplifier to an electric-circuit unit (R\&K CDE1500-KU) which is developed for this experiment. This unit includes a Bias-T, impedance adjuster, and a power detector which converts power of 
radio-frequency signal to DC voltage. Total gain from the parabola to the power detector is 117.7 $\mathrm{dB}$.

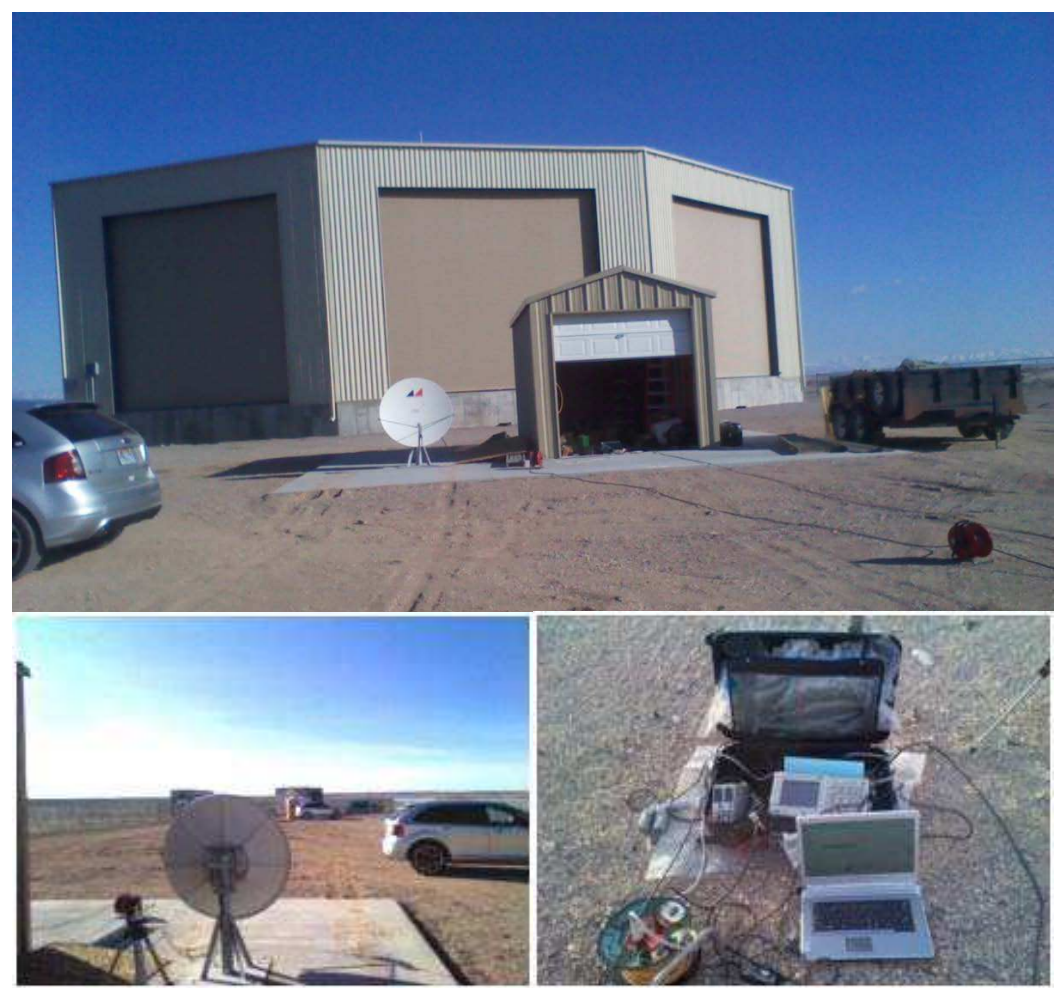

Figure 3: Photographs of observation system installed at TA observatory. The upper panel shows the parabola located front of the FD building. The lower-left panel shows the parabola seen from behind. The ELS is visible $88 \mathrm{~m}$ away from the parabola. The lower-right panel shows the data-acquisition system. The entire system is packed in a suitcase so that it may be transferred by commercial air travel from the laboratory to the observation site.

The sensitivity and gain of this observation system was calibrated piece by piece by several measurements. For example, the sensitivity of the parabola was measured in an anechoic room in the Microwave Energy Transmission Laboratory in Kyoto University [5]. A microwave signal at $12.5 \mathrm{GHz}$ was transferred into the feed horn from a network analyzer and reflected from the parabola. A two-dimensional radio scanner measured the near field to estimate the antenna pattern and the gain. The estimated antenna gain is $42.7 \mathrm{~dB}$, which is consistent with the catalog value as shown Figure 2.

This observation system was developed to search microwave from EAS on a building of Konan University which is located on Kobe Japan. Avoiding strong background noises below $10 \mathrm{GHz}$ from Kobe city, $12.5 \mathrm{GHz}$ band was selected to be measured [6]. Same detector was built on the TA observatory to measure microwave from the electron beam emitted from the ELS.

The ELS was constructed at the TA observatory to calibrate the FDs that detect ultra-violet photons from air showers [7]. The ELS emits up to $10^{9}$ electrons with energy of $40 \mathrm{MeV}$ as a pulse beam of $20 \mathrm{~ns} \sim 1 \mu \mathrm{s}$ in width. For our measurement the pulse was shaped as triangular of which the base is $20 \mathrm{~ns}$ and total charge of the beam was adjusted to be $100 \mathrm{pC}$. The electron beam was emitted into the atmosphere vertically. Total energy deposit in the atmosphere by the electron beam 
at $20 \mathrm{~m}$ above ground level is $1.4 \times 10^{15} \mathrm{eV} /\left(\mathrm{g} / \mathrm{cm}^{2}\right)$ which is equivalent to that by an air shower with an energy of $10^{18} \mathrm{eV}$ at its maximum.
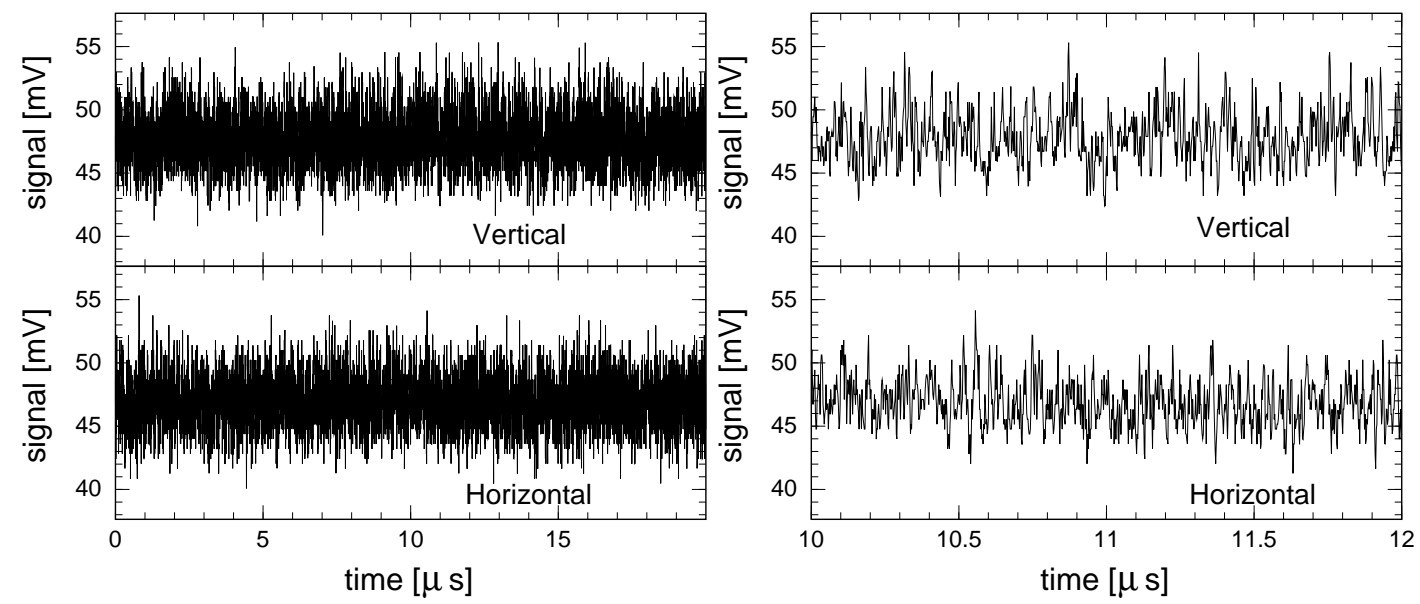

Figure 4: Typical output-signal waveform from power detector, as measured by oscilloscope. Vertically and horizontally polarized signals were measured. Left panels show $20 \mu$ s long waveform and right panels show the same signal but from 10 to $12 \mu \mathrm{s}$. A negative pulse from 11.31 to $11.34 \mu \mathrm{s}$ is expected but no clear pulse is seen.

The parabola was fixed on a concreate pad which was located between the ELS and the FD as shown in Figure 3. Elevation angle of the parabola was $15^{\circ}$ and the distance between the parabola and the electron beam is $88.0 \mathrm{~m}$. Because the field of view of the parabola at $12.5 \mathrm{GHz}$ is $1.22 \times$ $\lambda / 1.2 \mathrm{~m}=1.40^{\circ}$, the beam length in the field of view was $2.23 \mathrm{~m}$.

Figure 4 shows a typical output-signal waveform from the power detector recorded by the oscilloscope. Trigger signal was sent from the ELS to the oscilloscope. The signal is digitized with $2 \mathrm{~ns}$ sampling during $20 \mu$ s around a trigger signal. The microwave signal from the electron beam is expected to begin at $11.31 \mu \mathrm{s}$ as a negative pulse with $27.4 \mathrm{~ns}$ in width. In case of MBR, such negative pulse is expected in both of the horizontal and vertical polarizations. No significant signal is seen in this data. 1797 beams were measured. Average value of the DC voltage of the 1797 events is estimated as $47 \mathrm{mV}$. This value corresponds to $8.33 \times 10^{-21}\left[\mathrm{Wm}^{-2} \mathrm{~Hz}^{-1}\right]$ of the intensity of the microwave signal at the parabola. Considering statistical errors, a preliminary upper limit on the microwave intensity $88 \mathrm{~m}$ from the beam can be calculated as $9.4 \times 10^{-21}\left[\mathrm{Wm}^{-2} \mathrm{~Hz}^{-1}\right]$ with $95 \%$ confidence level. To compare with the previous measurement, the upper limit at 0.5 $\mathrm{m}$ from the beam can be estimated by a simple scaling as $2.91 \times 10^{-16}\left[\mathrm{Wm}^{-2} \mathrm{~Hz}^{-1}\right]$. This upper limit is close to that found in the previous measurements in Ref. [4], but these results cannot be compared directly because the experiments were configured differently (e.g., detector bandwidths and the reference beam lengths differ).

\section{Summary}

We studied the isotropic microwave radiation from air showers using an ELS. This radiation was proposed for UHECR research in [4], which reports detection of the MBR using electron 
accelerators. Motivated by this results, we attempted to detect $12.5 \mathrm{GHz}$ isotropic microwave radiation from electron beams by using a $1.2 \mathrm{~m}$ diameter parabola. An ELS was used to generate a $20 \mathrm{~ns}$ wide pulsed beam of $40 \mathrm{MeV}$ electrons. Each pulse contained electrons with $100 \mathrm{pC}$ of total charge. The energy deposited in the atmosphere by this electron beam is equivalent to that deposited near maximum of an air shower with energy of approximately $10^{18} \mathrm{eV}$. The parabola was positioned $88 \mathrm{~m}$ from the electron beam. No significant signal was detected. Based on this results, a preliminary upper limit on the intensity of the isotropic emission of $12 \mathrm{GHz}$ microwave from an air shower with an energy equivalent of $10^{18} \mathrm{eV}$ at $0.5 \mathrm{~m}$ from the shower axis was estimated as $2.91 \times 10^{-16}\left[\mathrm{Wm}^{-2} \mathrm{~Hz}^{-1}\right]$ with $95 \%$ confidence level.

\section{Acknowledgments}

The authors thank the Telescope Array collaboration for their help. The detectors were calibrated using the facilities at the Microwave Energy Transmission Laboratory, Research Institute for Sustainable Humanosphere. This work is supported by the Ministry of Education, Culture, Sports, Science and Technology, Japan, by a Grant-in-Aid for Scientific Research (B), 24340059, 2012 and a Grant-in-Aid for Challenging Exploratory Research, 21654036, 2009. It also partly supported by the Inter-University Research Program of the Institute of Cosmic Ray Research, University of Tokyo.

\section{References}

[1] J. Abraham et al. (The Pierre Auger Collaboration), NIMA 523 (2004) 50. http://www.auger.org.

[2] J. Abu-Zayyad, et al., NIMA 689 (2012) 87. http://www.telescopearray.org.

[3] The Pierre Auger, Telescope Array Collaborations, ApJ, 794 (2014) 172.

[4] P. W. Gorham et al., Phys. Rev. D 78 (2008) 032007.

[5] http://www.rish.kyoto-u.ac.jp/English/METLAB/index.html.

[6] T. Yamamoto, et al., Proc. of 33th ICRC (2013)

[7] T. Shibata et al., NIMA 597 (2008) 6166. 